\title{
Fidelity in topological quantum phases of matter
}

\author{
Silvano Garnerone, ${ }^{1, \text { f }}$ Damian Abasto, ${ }^{1}$ Stephan Haas, ${ }^{1}$ and Paolo Zanardi ${ }^{1, \oplus^{\dagger}}$ \\ ${ }^{1}$ Department of Physics and Astronomy, Center for Quantum Information Science\& Technology, \\ University of Southern California, Los Angeles, CA 90089
}

(Dated: December 3, 2018)

\begin{abstract}
Quantum phase transitions that take place between two distinct topological phases remain an unexplored area for the applicability of the fidelity approach. Here, we apply this method to spin systems in two and three dimensions and show that the fidelity susceptibility can be used to determine the boundary between different topological phases particular to these models, while at the same time offering information about the critical exponent of the correlation length. The success of this approach relies on its independence on local order parameters or breaking symmetry mechanisms, with which non-topological phases are usually characterized. We also consider a topological insulator/superconducting phase transition in three dimensions and point out the relevant features of fidelity susceptibility at the boundary between these phases.
\end{abstract}

PACS numbers: 03.65.Vf, 03.67.-a, 64.70.Tg, 24.10.Cn

\section{INTRODUCTION}

In recent years topological phases have been intensively studied in condensed matter systems. These exotic states appear in different contexts, such as fractional quantum Hall physics, spin liquids and topological insulators [1, 2, 3, 4, 5, 6, 7]. Their understanding is relevant to topological quantum computation, which provides the paradigm to store and manipulate information in topologically non-trivial systems [8]. In a seminal work [3] Kitaev introduced a spin model which can be exactly solved using a mapping to Majorana fermions coupled to a static $\mathbb{Z}_{2}$ gauge field ( [9], see also Ref. [10] for a perturbative approach). Generalizations of the Kitaev model with respect to lattice geometry, spatial dimension and local Hilbert space dimension have appeared recently [6, 11, 12]. In some regions of parameter space these models have nontrivial topological properties which cannot be described by any local order parameter. This prevents the direct applicability of the Landau-Ginzburg paradigm for the study of their phase transitions. Alternative ways of understanding criticality in systems with no local order parameter have been suggested in connection to quantum information. In particular, entanglement entropy (for a review see Ref.[13]) and the fidelity approach [14, 15, 16, 17, 18] have attracted a lot of attention (for a review see Ref.[19]). The reason why these two quantities can describe topological phases or determine their boundaries is due to the fact that they depend only on the properties of the ground state of the system and do not require a priori knowledge of any order parameter.

In this paper we shall focus on the fidelity approach to topological phase transitions [20, 21, 22]. Much work has been done in understanding the nature of the phases in the Kitaev honeycomb model and, in particular, its fidelity has been studied for the first time in Refs. [23, 24]. The present work aims to provide a more general understanding of fidelity in topo-

\footnotetext{
*Electronic address: garneron@ usc.edu

${ }^{\dagger}$ Also at Institute for Scientific Interchange, Viale Settimio Severo 65, I10133 Torino, Italy
}

logical phase transitions. For this porpuse we consider twoand three-dimensional extensions of the Kitaev honeycomb model. The models we chose differ from Kitaev's model in the geometry of the lattice, the interactions or the dimension of the ambient space and of the local Hilbert space.

\section{MODELS AND METHOD}

The dramatic changes in a many-body ground state $\left|\Psi_{0}(\lambda)\right\rangle$ across a quantum phase transition can be captured by the fidelity $F=\left|\left\langle\Psi_{0}(\lambda) \mid \Psi_{0}(\lambda+\delta \lambda)\right\rangle\right|$ between two ground states corresponding to slightly different values of the set of parameters defining the Hamiltonian $H(\{\lambda\})$. Alternatively, one can use the fidelity susceptibility $\chi=\lim _{\delta \lambda \rightarrow 0} \frac{-2 \ln F}{\delta \lambda^{2}}$ [25]. The fidelity quantifies how different two quantum states are. Given the drastic changes that take place across a quantum phase transition, one should expect the fidelity for nearby states to exhibit a drop across the boundary phase, signaling an enhanced distinguishability. Indeed, while in the thermodynamic limit the fidelity (susceptibility) has a drop (divergence) at the quantum critical point, for finite size systems this behavior is translated in the fidelity susceptibility being extensive away from criticality, while superextensive at criticality when the operator driving the transition is sufficiently relevant [18]. Moreover, a scaling analysis of the fidelity susceptibility can be performed to extract the critical exponent $\nu$ of the correlation length $\xi$.

Here we apply this tool from quantum information theory to study the quantum phase transition between two distinct topological phases in three models: mosaic models, Kitaev's honeycomb model with an external magnetic field, and a 3D model exhibiting a transition between two distinct non-abelian topological phases. Let us first review them very briefly.

An elegant way to look at the Kitaev model as a special instance of a more general class of models is provided by the mosaic classification [12]. Mosaic models are defined on twodimensional Bravais lattices constructed with a trivalent vertex building block, constrained by translational and rotational symmetry. The trivalent vertex is the local border between three polygons (see Fig 1 ). The number of edges of the three 


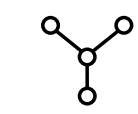

Trivalent block

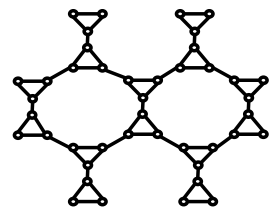

(3-12-12) model
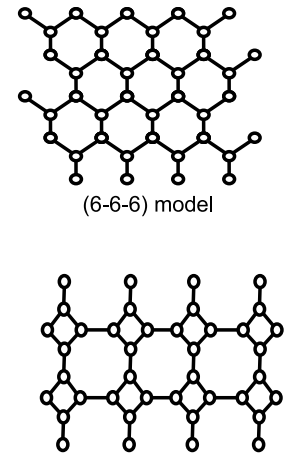

(4-8-8) model
FIG. 1: The trivalent vertex and the three different mosaic models constructed with it, which are considered in the present work.

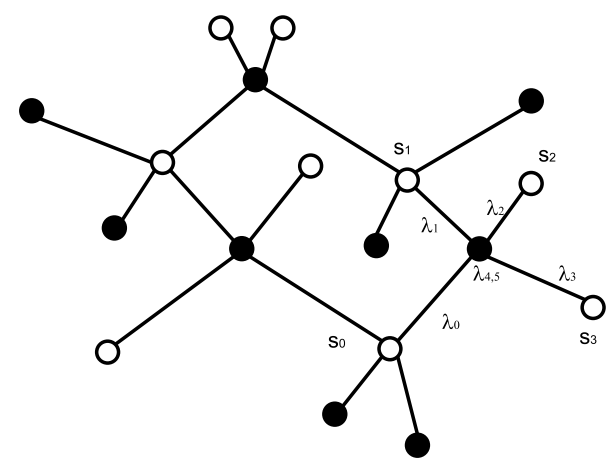

FIG. 2: The diamond lattice used in the three-dimensional extension of the Kitaev model.

polygons $\left(e_{1}, e_{2}, e_{3}\right)$ specifies the mosaic model and only four possible such lattices are allowed: $(6-6-6)$, corresponding to the Kitaev model; $(3-12-12)$, corresponding to the model studied in [6], $(4-6-12)$ and $(4-8-8)$.

The Hamiltonian of all mosaic spin models is given by

$$
H=-\sum_{u=x, y, z} J_{u} \sum_{(i, j) \in S(u)} \sigma_{i}^{u} \sigma_{j}^{u},
$$

where $S(u)$ is the set of edges in the $u$-direction. At every site the Hilbert space associated with the spin-1/2 particle is identified with a two-dimensional physical subspace $\mathcal{M}$ of a fourdimensional Fock space $\tilde{\mathcal{M}}$, introduced to solve the model. The Pauli operators acting on $\mathcal{M}$ are represented by four Majorana operators on $\tilde{\mathcal{M}}: \sigma^{x}=i b^{x} c, \sigma^{y}=i b^{y} c$ and $\sigma^{z}=i b^{z} c$, with $\alpha^{2}=1, \alpha \beta=-\beta \alpha$, for $\alpha, \beta \in\left\{b^{x}, b^{y}, b^{z}, c\right\}$, and $\alpha \neq \beta$. The physical subspace is obtained through a projection: $|\psi\rangle \in \mathcal{M} \Longleftrightarrow D|\psi\rangle=|\psi\rangle$, with $D=i b^{x} b^{y} b^{z} c$. The
Hamiltonian (1) can be rewritten as $H=\frac{1}{2} \sum_{i, j} \hat{A}_{i, j} c_{i} c_{j}$, with the operators $\hat{A}_{i j}=i J_{u} \hat{Z}_{i j}$, where $\hat{Z}_{i j}=i b_{i}^{u} b_{j}^{u}$ and $(i, j) \in S(u)\left(\hat{A}_{i j}=0\right.$ otherwise). The operators $\hat{Z}_{i j}$ commute with each other and with the Hamiltonian, so the space of the multi-spin system $\mathcal{L}$ can be decomposed as a direct sum $\mathcal{L}=\bigoplus_{Z} \mathcal{L}_{Z}$, where each sector is indexed by a set of eigenvalues $\left\{z_{i j}= \pm 1 \mid(i, j) \in S(u), u=x, y, z\right\}$ of the operator $\hat{Z}_{i j}$. Within each sector, the Hamiltonian reduces to a quadratic form in the Majorana operators $c_{i}$. The couplings of the Hamiltonian depend on a choice of vortex configuration, given by the eigenvalues of the plaquette operators $W_{p\left(n_{i}\right)}=-\prod_{i, j \in \partial p\left(n_{i}\right)} Z_{i j}$, one for each of the $n_{i}$ isogons $\left(\partial p\left(n_{i}\right)\right.$ is the set of links belonging to plaquette $\left.p\left(n_{i}\right)\right)$. The operators $W_{p\left(n_{i}\right)}$ commute with each other and with the Hamiltonian and since $W_{p\left(n_{i}\right)}^{2}=1$, their eigenvalues are $w_{p}= \pm 1$. A plaquette with $w_{p}=+1$ is a vortex free plaquette, while $w_{p}=-1$ corresponds to a vortex. In the following, we restrict ourselves to the vortex free sector, with a choice of $z_{i j}$ that preserves translational symmetry. The ground state energy is at a minimum for this configuration [26].

Another modification of the Kitaev model that preserves its topological nature is the addition of a three-spin interaction of the form

$$
H=-\sum_{u=x, y, z} J_{u} \sum_{(i, j) \in S(u)} \sigma_{i}^{u} \sigma_{j}^{u}-K \sum_{i, j, k} \sigma_{i}^{x} \sigma_{j}^{y} \sigma_{k}^{z},
$$

where the K-term is obtained through a perturbative expansion of a weak (Zeeman) magnetic field $V=\mathbf{h} \cdot \sigma$ [27]. In this case $K \sim \frac{h_{x} h_{y} h_{z}}{J^{2}}$ and this Hamiltonian is assumed to approximate the one with a Zeeman term when $K \ll J$, with $J_{u}=J$. The inclusion of this magnetic perturbation to the original model generates a topological phase with nonabelian anyonic excitations in the regime $\left|J_{z}\right| \leq\left|J_{x}\right|+\left|J_{y}\right|,\left|J_{y}\right| \leq$ $\left|J_{z}\right|+\left|J_{x}\right|,\left|J_{x}\right| \leq\left|J_{y}\right|+\left|J_{z}\right|$; while outside this region the excitations remain abelian.

Recently analogues of the Kitaev model on threedimensional lattices have been constructed [11, 28]. In the present work we focus on the model introduced in Ref. [11] since it presents quantum phase transitions between distinct non-trivial topological phases that, to our knowledge, have never been investigated with the fidelity approach. The model is defined on a diamond lattice and has a four-dimensional local Hilbert space (see Fig,2). The Hamiltonian is the following

$$
H=-\sum_{u=0}^{3} J_{u} \sum_{(i, j) \in S(u)} \sigma_{j}^{u} \sigma_{k}^{u}\left(\tau_{j}^{x} \tau_{k}^{x}+\tau_{j}^{z} \tau_{k}^{z}\right),
$$

where $\sigma$ and $\tau$ are Pauli spin matrices associated to the two local spin-1/2 degree of freedoms at each point in the lattice. As shown in [11] Hamiltonian (3) can be mapped to a free Majorana Hamiltonian which, in its ground-state sector, is given by

$$
H=i \sum_{u=0}^{3} J_{u} \sum_{(i, j) \in S(u)}\left(\lambda_{j}^{4} \lambda_{k}^{4}+\lambda_{j}^{5} \lambda_{k}^{5}\right),
$$


and $\lambda_{i}^{4,5}$ are Majorana fermions introduced in the representation of the Pauli spin matrices (see Ref. [11] for details), analogously to the previous model Hamiltonians. The addition of next-nearest-neighbour interactions into (4) allows for the presence of distinct non-trivial topological phases in the phase diagram, as we shall see in the next section.

The spin realization of all the above models can be mapped onto a free Majorana fermion Hamiltonian

$$
H(A)=\frac{i}{4} \sum_{i, j} A_{i, j} c_{i} c_{j},
$$

where $A_{i, j}$ encodes the lattice structure and the parameters of the model. Once a unit cell of size $s$ has been chosen the Hamiltonian can be written in direct space as

$$
H(A)=\frac{i}{4} \sum_{n, \nu ; m, \mu} A_{n, \nu ; m, \mu} c_{n, \nu} c_{m, \mu}
$$

where $n$ and $m$ are the positions of the unit cells in the lattice and $\mu$ and $\nu$ are the positions of the vertices inside the unit cell. Due to translational invariance $A$ depends only on $\nu, \mu$ and $m-n$. Using a Fourier transform, we obtain

$$
H=\sum_{\mathbf{k}} \boldsymbol{\Psi}^{\dagger}(\mathbf{k}) \mathcal{H}(\mathbf{k}) \Psi(\mathbf{k}),
$$

with $\Psi_{\alpha}(\mathbf{k})=1 / \sqrt{N} \sum_{\mathbf{r}} e^{-i \mathbf{k} \cdot \mathbf{r}} c_{\mathbf{r}, \alpha}$ a complex fermion, $N=$ $s L^{d}$ the system size, $L$ the number of unit cells in one direction of the d-dimensional lattice and $s$ the size of the unit cell, with $\alpha \in\{1,2, . ., s\}$. Then in general we have to deal with a free-fermion model characterized by $s$ different bands. The ground-state is obtained filling the Fermi sea, where all the levels with negative energy $\epsilon_{\beta}(\mathbf{k})<0$ are occupied,

$$
\left|\Psi_{0}\right\rangle \equiv \prod_{\beta, \mathbf{k}} b_{\beta}^{\dagger}(\mathbf{k})|0\rangle
$$

if $\epsilon_{\beta}(\mathbf{k})<0 . \quad b_{\beta}^{\dagger}(\mathbf{k}) \equiv \boldsymbol{\Psi}^{\dagger}(\mathbf{k}) \cdot \mathbf{V}_{\beta}(\mathbf{k})$, where $\mathbf{V}_{\beta}(\mathbf{k})$ is the eigenvector associated to the $\epsilon_{\beta}(\mathbf{k})$ eigenvalue of $\mathcal{H}(\mathbf{k})$. The matrix $U(\mathbf{k})$ diagonalizing $\mathcal{H}(\mathbf{k})$ has as column vectors $V_{\beta}(\mathbf{k})$. The spectrum of the single-body Hamiltonian $\mathcal{H}(\mathbf{k})$ is symmetric around zero.

The k-component of the many-body groundstate $\left|\Psi_{0}\right\rangle=$ $\prod_{\mathbf{k}}\left|\Psi_{0}\right\rangle_{\mathbf{k}}$ in first quantization is given by a functional Slater determinant. The $j$-th component of the eigenstate corresponding to the $\beta$-th band is represented by $V_{j, \beta}(\mathbf{k})$. Denoting with $s_{n}$ the number of negative single particle energy bands, the first quantized wave function at fixed $\mathbf{k}$ is the Slater determinant of $s_{n}$ particles that can occupy $s_{n}$ bands

$$
\left\langle j_{1}, \ldots, j_{s_{n}} \mid \Psi_{0}\right\rangle_{\mathbf{k}}=\frac{1}{\sqrt{s_{n} !}}\left|\begin{array}{ccc}
V_{j_{1}, 1}(\mathbf{k}) & \cdots & V_{j_{1}, s_{n}}(\mathbf{k}) \\
\vdots & \ddots & \vdots \\
V_{j_{s_{n}}, 1}(\mathbf{k}) & \cdots & V_{j_{s_{n}}, s_{n}}(\mathbf{k})
\end{array}\right|
$$

with $j_{i} \in\{1,2, \ldots, s\}$. For these particular models, the fidelity corresponds to the product over all $\mathbf{k}$ of the absolute value of the overlap of two Slater determinants at different parameter values $\left\|\left\langle\tilde{\Psi}_{0} \mid \Psi_{0}\right\rangle\right\|=\bigotimes_{\mathbf{k}}\left\|\left\langle\tilde{\Psi}_{0} \mid \Psi_{0}\right\rangle_{\mathbf{k}}\right\|$ with

$$
\begin{array}{r}
\left\langle\tilde{\Psi}_{0} \mid \Psi_{0}\right\rangle_{\mathbf{k}}=\frac{1}{s_{n} !} \sum_{j_{1}, \ldots, j_{s_{n}}}\left|\begin{array}{ccc}
\tilde{V}_{j_{1}, 1}(\mathbf{k}) & \cdots & \tilde{V}_{j_{1}, s_{n}}(\mathbf{k}) \\
\vdots & \ddots & \vdots \\
\tilde{V}_{j_{n}, 1}(\mathbf{k}) & \cdots & \tilde{V}_{s_{s_{n}}, s_{n}}(\mathbf{k})
\end{array}\right|^{*} \\
\times\left|\begin{array}{ccc}
V_{j_{1}, 1}(\mathbf{k}) & \cdots & V_{j_{1}, s_{n}}(\mathbf{k}) \\
\vdots & \ddots & \vdots \\
V_{s_{s_{n}}, 1}(\mathbf{k}) & \cdots & V_{j_{s_{n}}, s_{n}}(\mathbf{k})
\end{array}\right|
\end{array}
$$

The previous expression, using the properties of determinants, can be rewritten as (for a proof see pag.291 of Ref.[29])

$$
\left|\begin{array}{ccc}
\tilde{\mathbf{V}}_{1}^{*}(\mathbf{k}) \cdot \mathbf{V}_{1}(\mathbf{k}) & \cdots & \tilde{\mathbf{V}}_{1}^{*}(\mathbf{k}) \cdot \mathbf{V}_{s_{n}}(\mathbf{k}) \\
\vdots & \ddots & \vdots \\
\tilde{\mathbf{V}}_{s_{n}}^{*}(\mathbf{k}) \cdot \mathbf{V}_{1}(\mathbf{k}) & \cdots & \tilde{\mathbf{V}}_{s_{n}}^{*}(\mathbf{k}) \cdot \mathbf{V}_{s_{n}}(\mathbf{k})
\end{array}\right|
$$

The above formula will be used in the evaluation of fidelity for the models that we consider.

\section{RESULTS}

In this section we study how the fidelity susceptibility behaves for the mosaic models $(4-8-8)$ and $(3-12-12)$, for the model (2), and for the three-dimensional Kitaev model (3).

\section{A. Mosaic models}

Let us first focus on the $(4-8-8)$ mosaic model [30]. Choosing a four-site unit cell, a Fourier transform of the Hamiltonian gives

$$
H=\frac{1}{2} \sum_{\mathbf{k}} \Psi^{\dagger}(\mathbf{k}) \mathcal{H}(\mathbf{k}) \Psi(\mathbf{k})
$$

where

$$
\mathcal{H}(\mathbf{k})=\left(\begin{array}{cc}
J_{x} \sigma^{y} & -i J_{y} \sigma^{x}+i J_{z} \alpha \\
i J_{y} \sigma^{x}-i J_{z} \alpha^{\dagger} & J_{x} \sigma^{y}
\end{array}\right)
$$

$\Psi_{\mathbf{k}}^{\dagger}=\left(a_{\mathbf{k}, 1}^{\dagger}, a_{\mathbf{k}, 2}^{\dagger}, a_{\mathbf{k}, 3}^{\dagger}, a_{\mathbf{k}, 4}^{\dagger}\right)$ is the Fourier transform of the Majorana operators $\left\{\Psi_{i}\right\}_{i=1}^{4}, \alpha=$ $\operatorname{diag}\left[\exp \left(-i k_{2}\right),-\exp \left(i k_{1}\right)\right], k_{1}=\mathbf{k} \cdot \mathbf{n}_{1}, k_{2}=\mathbf{k} \cdot \mathbf{n}_{2}$, $\mathbf{n}_{1}=(1,0)$ and $\mathbf{n}_{2}=(0,1)$. The system size is $N=2 L^{2}$. This model presents a quantum phase transition between two gapped phases with abelian anyons when $J_{z}^{2}=J_{x}^{2}+J_{y}^{2}$. The phases are algebraically distinct, though they can be related by rotational symmetry.

For the $(3-12-12)$ mosaic model [6, 31], a Fourier transform with a six-unit cell renders the Hamiltonian in the form 


$$
\begin{aligned}
H & =\frac{i}{2}\left(-a_{\mathbf{k}, 1}^{\dagger} a_{\mathbf{k}, 2}-a_{\mathbf{k}, 1}^{\dagger} a_{\mathbf{k}, 3}+J e^{-i \mathbf{k} \cdot \mathbf{n}_{1}} a_{\mathbf{k}, 1}^{\dagger} a_{\mathbf{k}, 2}\right. \\
& +a_{\mathbf{k}, 2}^{\dagger} a_{\mathbf{k}, 3}-J e^{i \mathbf{k} \cdot \mathbf{n}_{2}} a_{\mathbf{k}, 1}^{\dagger} a_{\mathbf{k}, 2}-J a_{\mathbf{k}, 3}^{\dagger} a_{\mathbf{k}, 6} \\
& \left.-a_{\mathbf{k}, 4}^{\dagger} a_{\mathbf{k}, 5}-a_{\mathbf{k}, 4}^{\dagger} a_{\mathbf{k}, 6}-a_{\mathbf{k}, 5}^{\dagger} a_{\mathbf{k}, 6}\right)+\mathrm{hc}
\end{aligned}
$$

with $\mathbf{n}_{1}=(1 / 2, \sqrt{3} / 2)$ and $\mathbf{n}_{2}=(1 / 2,-\sqrt{3} / 2)$. The single particle Hamiltonian $A(\mathbf{k})$ is $6 \times 6$, and the system size is $N=6 L^{2}$. This system has a quantum phase transition at $J=\sqrt{3}$ from a topological phase with abelian anyons to one with nonabelian anyons, spontaneously breaking time reversal symmetry, without the need of an external magnetic field.

The numerical results for the fidelity susceptibility $\chi=$ $\lim _{\delta \lambda \rightarrow 0}-2 \ln F / \delta \lambda^{2}$ for the $(4-8-8)$ mosaic model are shown in Fig. 3 for different system sizes, taking $J_{x}=J_{y}=$ $1, J_{z} \in(1.21,1.61)$. We see that $\chi$ is an extensive quantity off criticality, while it is superextensive at the critical point $J_{z}^{c}=\sqrt{2}$.

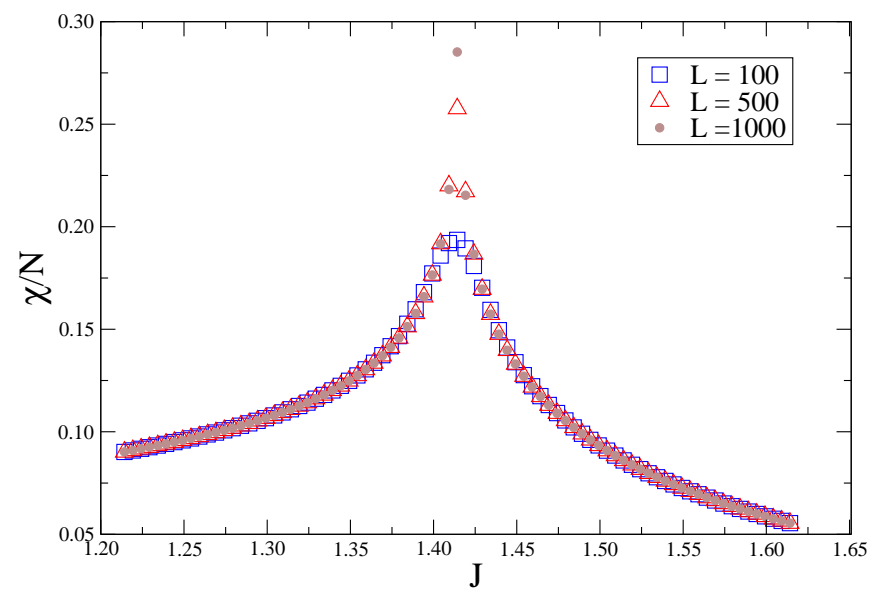

FIG. 3: (Color online). Fidelity susceptibility $\chi$ per site for the $(4-8-8)$ mosaic model around the quantum critical point $J_{z}=\sqrt{2}$, separating two topological phases $\left(J_{x}=J_{y}=1\right)$. $\chi$ shows extensive behavior off criticality, while it is superextensive at the critical point.

We perform finite-size scaling for the susceptibility around the critical point $J_{z}^{c}=\sqrt{2}$. For a finite sample size we denote the point at which $\chi$ is maximum as $\chi^{\max }$, evaluated at $J_{z}=$ $J_{z}^{\max }$. It scales like $\chi^{\max } / N \propto L^{\mu}$, with $\mu>0$, while in the thermodynamic limit $\chi / N \propto 1 /\left|J^{\prime}-J_{z}^{c}\right|^{\alpha}$. In Fig. 4(a) we plot $\log \chi^{\max }$ vs $\log L$, for system sizes $L \in[300,1000]$ in steps of 100 , with $\delta J=10^{-6}$. We obtain superextensive scaling given by $\mu=0.1523 \pm 0.0001$.

Furthermore, we perform a data collapse, using the scaling ansatz [32]

$$
\frac{\chi(J)}{\chi^{\max }} \simeq f\left(\frac{J_{z}-J_{z}^{\max }}{\sqrt{2}} L^{1 / \nu}\right) .
$$

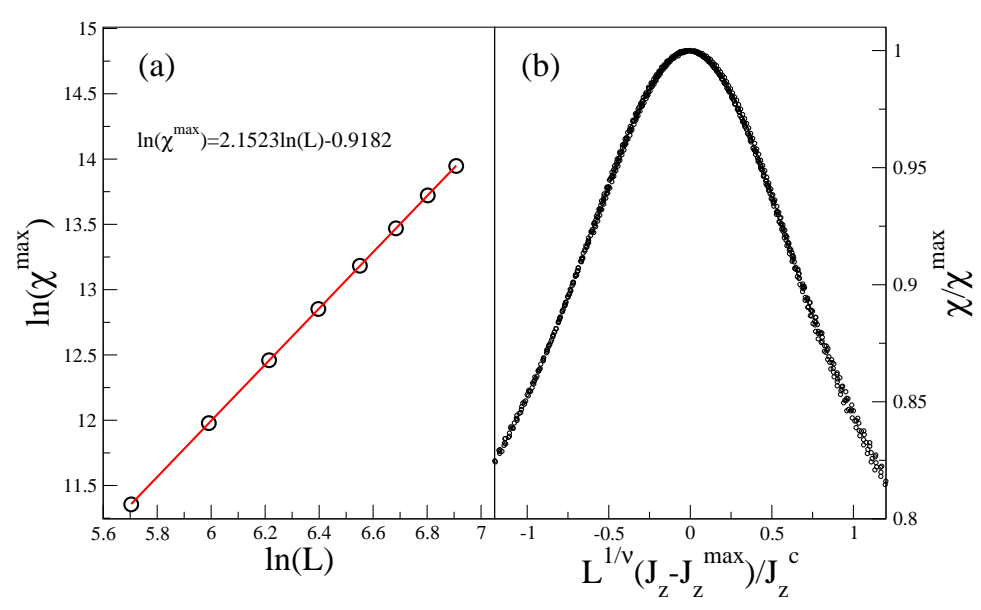

FIG. 4: (Color online). (a) Scaling analysis of the fidelity susceptibility at criticality for the $(4-8-8)$ mosaic model, for system sizes $L \in[300,1000]$ in steps of 100 , with $\delta J=10^{-6}$. We obtain that $\chi^{\max } / N \simeq L^{\mu}$, with $\mu=0.1523 \pm 0.0001$. (b) Data collapse for the fidelity susceptibility around the critical point $J_{z}^{c}=\sqrt{2}$. All curves collapse when plotted as a function of the dimensionless quantity $L^{1 / \nu}\left(J_{z}-J_{z}^{\max }\right) / J_{z}^{c}$, with critical exponent $\nu=1.105 \pm 0.070$

To quantify the extent of the collapse, we use a method similar to the one described in Ref.[33], obtaining the value $\nu=1.105 \pm 0.07$. From there, we conclude that $\alpha=\frac{\mu}{\nu}=$ $0.138 \pm 0.009$. The result for the data collapse is shown in Fig. 4 (b).

A similar analysis has been performed for the $(3-12-12)$ mosaic model [6], confirming that the fidelity is superextensive at criticality, with $\mu=0.155 \pm 0.009$ as seen on Fig. 5.

However, we have not been able to obtain a satisfactory data collapse for the $(3-12-12)$ mosaic model, probably due to numerical errors.

\section{B. Kitaev model with 3-spin interaction}

This model can be exactly diagonalized using the techniques we have summarized above. In particular, the Hamiltonian in equation (2) takes the form $H=\frac{1}{2} \sum_{i, j} \hat{A}_{i, j} c_{i} c_{j}$, with $\hat{A}_{i j}=i J_{u} \hat{Z}_{i j}+K \sum_{k} \hat{Z}_{i k} \hat{Z}_{j k}$, and for the vortex-free case, the spectral matrix in momentum space is

$$
\mathcal{H}(\mathbf{k})=\left(\begin{array}{cc}
g(\mathbf{k}) & i f(\mathbf{k}) \\
-i f^{*}(\mathbf{k}) & -g(\mathbf{k})
\end{array}\right)
$$

where $f(\mathbf{k})=J_{z}+J_{x} e^{i \mathbf{k} \cdot \mathbf{n}_{1}}+J_{2} e^{i \mathbf{k} \cdot \mathbf{n}_{2}}, g(\mathbf{k})=$ $2 K\left(\sin \mathbf{k} \cdot \mathbf{n}_{1}-\sin \mathbf{k} \cdot \mathbf{n}_{2}+\sin \mathbf{k} \cdot\left(\mathbf{n}_{2}-\mathbf{n}_{1}\right)\right)$, with $\mathbf{n}_{1}=$ $(1,0)$ and $\mathbf{n}_{2}=(0,1)$. The system size is $N=2 L^{2}$.

The inclusion of an external magnetic field gives rise to a transition between phases with abelian and nonabelian anyon 


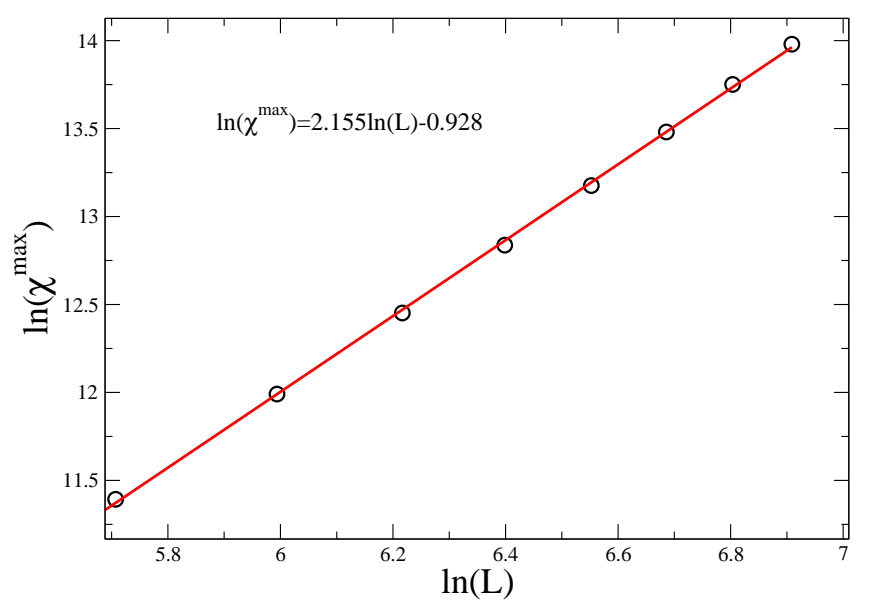

FIG. 5: (Color online). Scaling analysis of the susceptibility at criticality for the $(3-12-12)$ mosaic model, for $L \in[301,1001]$ in steps of 100 . The fidelity signals the quantum phase transition separating the two distinct topological phases at $J=\sqrt{3}$, behaving as $\chi^{\max } / N \simeq L^{\mu}$, with $\mu=0.155 \pm 0.009$

excitations, contained in the region $\left|J_{z}\right| \leq\left|J_{x}\right|+\left|J_{y}\right|,\left|J_{y}\right| \leq$ $\left|J_{z}\right|+\left|J_{x}\right|,\left|J_{x}\right| \leq\left|J_{y}\right|+\left|J_{z}\right|$ of the parameter space.

The numerical results for $\chi^{\max } / N \propto L^{\mu}$ for the honeycomb model with external magnetic field are shown in Fig. 6 (a), where we obtain $\mu=0.1735 \pm 0.0001$, signaling superextensive scaling at criticality. The corresponding data collapse is shown in Fig. 6 (b), with $\nu=1.10 \pm 0.05$ for the critical exponent of the correlation length, and $\alpha=\mu / \nu=$ $0.158 \pm 0.007$.

For the models we have considered so far we see that the fidelity susceptibility is able to detect the phase transition between distinct topological phases, besides providing useful information about the critical point itself (i.e. the exponent $\nu$ of the correlation length).

\section{3 dimensional model}

The Hamiltonian in equation (3) has lines of zeros in momentum space with a vanishing gap. The addition of next-nearest-neighbour interactions that preserve time reversal symmetry of the form

$$
\begin{aligned}
& H_{\mathrm{nnn}}^{z}=i \sum_{\mathrm{jk}} t^{z}\left(\lambda_{j}^{4} \lambda_{k}^{4}-\lambda_{j}^{5} \lambda_{k}^{5}\right) \\
& H_{\mathrm{nnn}}^{x}=i \sum_{\mathrm{jk}} t^{x}\left(\lambda_{j}^{4} \lambda_{k}^{5}+\lambda_{j}^{5} \lambda_{k}^{4}\right)
\end{aligned}
$$

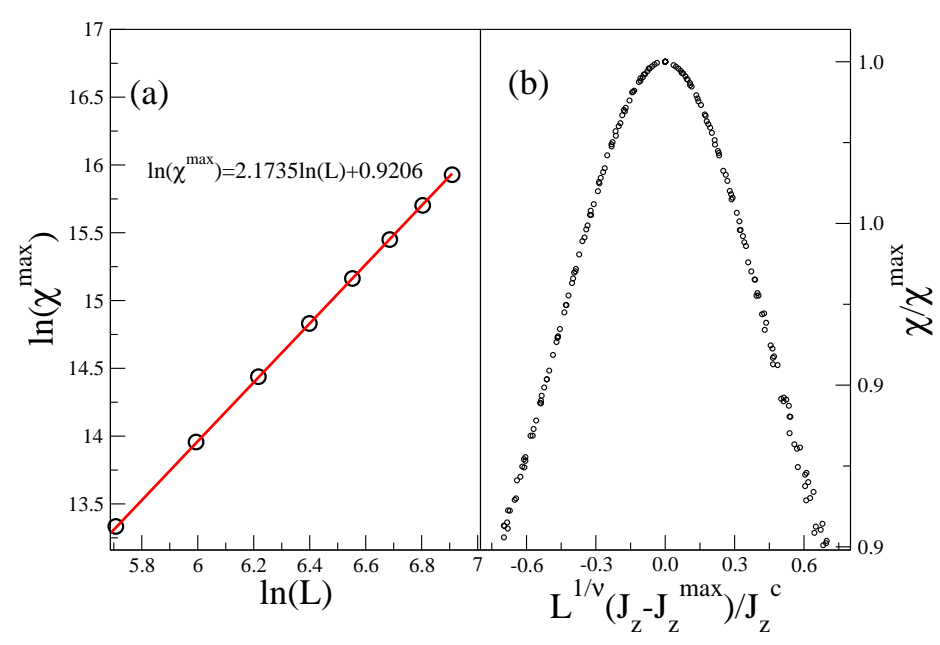

FIG. 6: (Color online). (a) Scaling analysis of the susceptibility for the Kitaev model with a weak Zeeman magnetic field, at the critical point $J_{z}=1 / 2$. The system size range is $L \in[301,1001]$ in steps of 100 , and the coupling $K=1 / 15$. The data implies that $\chi^{\max } / N \propto$ $L^{\mu}$, with $\mu=0.1735 \pm 0.0001$ at criticality. (b) Data collapse of the fidelity susceptibility $\chi$ around the critical point $J_{z}^{c}=1 / 2\left(J_{x}=\right.$ $\left.J_{y}=0.5\left(1-J_{z}\right)\right)$ and $K=1 / 15$. We obtain $\nu=1.10 \pm 0.05$ for the critical exponent of the correlation length.

removes the degeneracy [11]. In momentum space the Hamiltonian (4) with the above perturbations can be written as

$$
H=i \sum_{\mathrm{k}}\left(a_{-k}^{4}, a_{-k}^{5}, b_{-k}^{4}, b_{-k}^{5}\right) \mathcal{H}(k)\left(a_{k}^{4}, a_{k}^{5}, b_{k}^{4}, b_{k}^{5}\right),
$$

with $\mathcal{H}(k)$ given by

$$
\mathcal{H}(\mathbf{k})=\left(\begin{array}{cc}
\Theta(\mathbf{k}) & i \Phi(\mathbf{k}) \\
-i \Phi^{*}(\mathbf{k}) & -\Theta(\mathbf{k})
\end{array}\right) .
$$

Defining the three component vectors $s_{0}=1 / 4(-1,1,-1)$, $s_{1}=1 / 4(1,1,1), s_{2}=1 / 4(-1,-1,1)$ and $s_{3}=$ $1 / 4(1,-1,-1)$ (see Fig 2), the blocks of the matrix (20) are given by

$$
\begin{gathered}
\Phi(\mathbf{k})=\sum_{u=0}^{3} \sigma^{0} J_{u} e^{i \mathbf{k} \cdot s_{u}}, \\
\Theta(\mathbf{k})=\Theta^{x}(\mathbf{k}) \sigma^{x}+\Theta^{z}(\mathbf{k}) \sigma^{z}, \\
\Theta^{x}(\mathbf{k})=t^{x}\left[\sin \frac{k_{x}-k_{y}}{2}+\sin \frac{k_{y}-k_{z}}{2}+\sin \frac{k_{z}-k_{x}}{2}\right], \\
\Theta^{z}(\mathbf{k})=t^{z} \sin \frac{k_{y}+k_{z}}{2},
\end{gathered}
$$

with $\sigma^{0}$ the two-dimensional identity matrix and $\sigma^{x}, z$ the usual Pauli matrices. 
When all couplings $J$ are equal, there are still three degenerate points in the Brillouin zone at $(2 \pi, 0,0),(0,2 \pi, 0)$ and $(0,0,2 \pi)$. These degeneracies can be made massive by adding a small anisotropy in one of the couplings $J$. Following [11] we set $t^{x}=1, J_{2,3,4}=2$ and $J_{1}=2+\delta J_{1}$. The free parameters of the model are then $t^{z}$ and $\delta J_{1}$. The phase diagram presents three topologically distinct phases, distinguished by the winding number. For positive $\delta J_{1}$ and positive $t_{z}$ the winding number is +1 ; for positive $\delta J_{1}$ and negative $t_{z}$ the winding number is -1 , and it is zero in the negative $\delta J_{1}$ region [11]. There are two topological phase transitions at the two critical lines $\delta J_{1}=0$ and $t_{z}=0$.

We have studied this model using the fidelity approach and in the following we present the results for the scaling of the fidelity susceptibility around the two phase transitions of the model. In Fig 7 (a) we show the log-log plot of the fidelity susceptibility at the $t_{z}=0$ critical line, while Fig 7 (b) presents analogous results for the transition across the $\delta J_{1}=0$ line. In both cases the fidelity susceptibility has a peak at the critical point. The scaling analysis suggests that the fidelity susceptibility is extensive within the numerical precision that we could obtain from a first set of data. However, the numerical accuracy of this result cannot exclude a weak super-extensive scaling at the critical point. A more accurate analysis of this model will be presented in a future work.

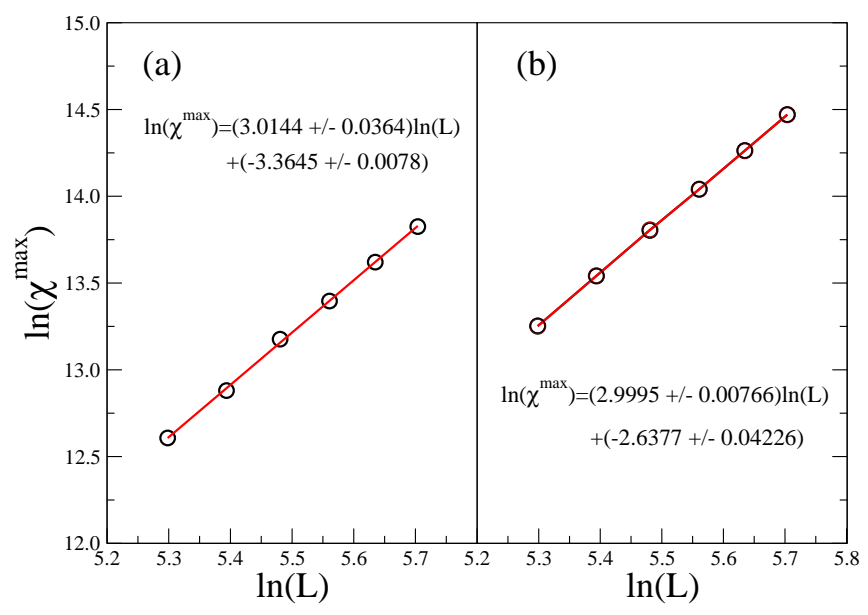

FIG. 7: (a) Log-log plot of the fidelity susceptibility at the critical line for $\delta J_{1}=1$, and linear system size $L \in[200,300]$ in steps of 20. (b) Log-log plot of the fidelity susceptibility at the critical line for $t_{z}=0.1$, and linear system size $L \in[200,300]$ in steps of 20

\section{CONCLUSIONS}

The fidelity approach proves to be a useful tool to detect and characterize quantum phase transitions which take place between distinct topological phases. We have applied it to the family of mosaic models which presents a transition between topological phases with abelian and nonabelian anyons, as well as the Kitaev model with an external magnetic field, where a transition from an abelian to a nonabelian phase takes place. We were able to show superextensive scaling of the fidelity susceptibility at criticality, characterizing its behavior in the thermodynamic limit. We also extracted the critical exponent of the correlation length for these models. Moreover, we studied the transition between a topological insulator and a topological superconductor in a three-dimensional diamond lattice, where the method still detects the boundary between different phases. In this case, superextensivity of the fidelity susceptibility turns out to be more difficult to be established. This is probabily due to the increased dimensionality which weakens the features of the quantum phase transitions.

We have thus extended the applicability of the fidelity approach to the boundary that separates different topological phases and proved its usefulness in this context as well. Since it is not always clear how to detect and distinguish different topological phases of matter we believe it is important to check the validity of a method based solely on the geometrical properties of the groundstate, and that does not require the introduction of an order parameter.

\section{ACKNOWLEDGEMENTS}

We thank K. Shtengel for pointing us reference [6] and H. Yao for helpful discussions. Computation for the work described in this paper was supported by the University of Southern California Center for High Performance Computing and Communications. We acknowledge financial support by the National Science Foundation under grant DMR-0804914.
[1] X.-G. Wen, Int. J. Mod. Phys. B 4, 239 (1990); X.-G. Wen and Q. Niu, Phys. Rev. B 41, 9377 (1990); X.-G. Wen, Phys. Rev. B 44, 2664 (1991).

[2] N. Read and S. Sachdev, Phys. Rev. Lett. 66, 1773 (1991).

[3] A. Y. Kitaev, Ann. Phys. 321, 2 (2006).

[4] D.J. Thouless, M. Kohmoto, M. P. Nightingale and M. den Nijs,
Phys. Rev. Lett. 49, 405 (1982).

[5] C. L. Kane and E. J. Mele, Phys. Rev. Lett. 95, 146802 (2005).

[6] H. Yao and S. A. Kivelson, Phys. Rev. Lett. 99, 247203 (2007).

[7] B. A. Bernevig, T. L. Hughes and S. Zhang, Science 314, 1757 (2006).

[8] C. Nayak, S. H. Simon, A. Stern,M. Freedman and S. Das 
Sarma, arXiv:0707.1889

[9] J. K. Pachos, Ann. of Phys. 6, 1254 (2006).

[10] J. Vidal, K.P. Schmidt and S. Dusuel, Phys. Rev. B 78, 245121 (2008).

[11] S. Ryu, arXiv:0811.2036

[12] S. Yang, D. L. Zhou and C. P. Sun, Phys. Rev. B, 76, 180404(R) (2007).

[13] L. Amico, R. Fazio, A. Osterloh and V. Vedral, Rev. Mod. Phys. 80, 517 (2008).

[14] P. Zanardi and N. Paunković, Phys. Rev. E 74, 031123 (2006).

[15] H.-Q. Zhou and J. P. Barjaktarevič, arXiv:cond-mat/0701608, H. -Q. Zhou, J. -H. Zhao and B.Li, arXiv:0704.2940, H.-Q. Zhou, arXiv:0704.2945

[16] P. Zanardi, P. Giorda and M. Cozzini, Phys. Rev. Lett. 99, 100603 (2007).

[17] P. Zanardi, M. Cozzini and P. Giorda, J. Stat. Mech. L02002 (2007).

[18] L. Campos Venuti and P. Zanardi, Phys. Rev. Lett. 99, 095701 (2007).

[19] S.-J. Gu, arXiv:0811.3127

[20] A. Hamma, W. Zhang, S. Haas and D.A. Lidar, Phys. Rev. B 77, 155111 (2008).

[21] D. F. Abasto, P. Zanardi, Phys. Rev. A 79, 012321 (2009).
[22] D. F. Abasto, A. Hamma, P. Zanardi, Phys. Rev. A 78, 010301(R), 2008.

[23] S. Yang, S. -J. Gu, C. -P. Sun and H. -Q. Lin, Phys. Rev. A, 78, 012304 (2008).

[24] J.-H. Zhao and H.-Q. Zhou, arXiv:cond-mat/0803.0814v1.

[25] W. -L. You, Y. -W. Li and S. -J. Gu, Phys. Rev. E 76, 022101 (2007).

[26] E. H. Lieb, Phys. Rev. Lett. 73, 2158 (1994).

[27] V. Lahtinen, G. Kells, A. Carollo, T. Stitt, J. Vala and J. K. Pachos, Ann. Phys. 323, 2286 (2008).

[28] C. Wu, D. Arovas and H.-H Hung, arXiv:0811.1380v2

[29] J.C.Slater, Quantum Theory of Atomic Structure (McGrawHill, London, 1960), Vol.1, pag.291.

[30] S. Yang, D. L. Zhou and C. P. Sun, Phys. Rev. B, 76, 180404(R) (2007).

[31] S. Dusuel, K.P. Schmidt, J. Vidal and R.L. Zaffino, Phys. Rev. B 78, 125102 (2008).

[32] J. G. Brankov, D. M. Danchev and N. S. Tonchev, Theory of Critical Phenomena in Finite-Size Systems, World Scientific, 2000.

[33] S. M. Bhattacharjee and F. Seno, J. Phys. A: Math. Gen. 34, 6375-6380, (2001). 